\title{
Machine Learning in Medicine:
}

\author{
Opening the New Data Protection Black Box
}

\begin{abstract}
Agata Ferretti, Manuel Schneider and Alessandro Blasimme*
Artificial intelligence (AI) systems, especially those employing machine learning methods, are often considered black boxes, that is, systems whose inner workings and decisional logics remain fundamentally opaque to human understanding. In this article, we set out to clarify what the new General Data Protection Regulation (GDPR) says on profiling and automated decision-making employing opaque systems. More specifically, we focus on the application of such systems in the domain of healthcare. We conducted a conceptual analysis of the notion of opacity (black box) using concrete examples of existing or envisaged medical applications. Our analysis distinguishes among three forms of opacity: (i) lack of disclosure, (ii) epistemic opacity, and (iii) explanatory opacity. For each type of opacity, we discuss where it originates from, and how it can be dealt with according to the GDPR in the context of healthcare. This analysis can offer insights regarding the contested issue of the explainability of AI systems in medicine, and its potential effects on the patient-doctor relationship.
\end{abstract}

Keywords: Artificial Intelligence, Machine Learning, Black Box, Medicine, GDPR, Transparency

\section{Introduction}

Artificial intelligence ${ }^{1}$ (AI) is the talk of the town. In recent years, we have witnessed a growing interest for the rapid development and application of AI systems in virtually any domain of human activity. Even objects of ordinary use such as thermostats, smartphones and cars now employ AI systems to process data and automatically perform an increasing number of tasks. In particular, some fields of AI

DOI: $10.21552 / \mathrm{edpl} / 2018 / 3 / 10$

* Agata Ferretti, equal contributor, PhD candidate at the Health Ethics and Policy Lab, ETH Zurich. Manuel Schneider, equal contributor, PhD candidate at the Health Ethics and Policy Lab, ETH Zurich. Dr Alessandro Blasimme, Senior Researcher in Bioethics at the Health Ethics and Policy Lab, ETH Zurich. For correspondence: <alessandro.blasimme@ hest.ethz.ch>.

1 According to a widespread origin story, the term artificial intelligence (Al) was first introduced by mathematician and computer scientist John McCarthy in an application proposal for a Summer workshop held at Dartmouth College in 1956, which is considered the event that started Al as a scientific field (see Nils J Nilsson, The quest for artificial intelligence (Cambridge University Press 2009)). For more background on the research field and in-depth technical readings about Al we recommend the following two books: Stuart J Russell and Peter Norvig, Artificial intelligence: a modern approach (Pearson Education Limited 2016) and lan Goodfellow, Yoshua Bengio and Aaron Courville, Deep Learning (MIT Press 2016). such as machine learning (ML) are attracting attention in both academic circles and the popular press. These systems have the ability to learn, on their own or through human supervision, how to perform a task such as, for instance, recognizing a road sign. But AI systems are also employed to automate certain judicial decisions, for instance to help judges predict the risk of recidivism, ${ }^{2}$ or in the financial industry to aid decisions about loans ${ }^{3}$ or insurance policies. $^{4}$

2 Dzonale A Andrews, James Bonta and J Stephen Wormith, 'The Recent Past and Near Future of Risk and/or Need Assessment' (2006) 52 Crime \& Delinquency 7.

3 Kori Hale, 'Al Challenging Bank Lending Practices' (Forbes, 2018) <https://www.forbes.com/sites/korihale/2018/05/22/ai -challenging-banks-lending-practices/\#77f499657e0e> accessed 10 July 2018; Jon Walker, 'Artificial Intelligence Applications for Lending and Loan Management' (TechEmergence, 2018) $<$ https://www.techemergence.com/artificial-intelligence -applications-lending-loan-management/> accessed 10 July 2018.

4 Executive Office of the President and others, Big data: A report on algorithmic systems, opportunity, and civil rights (Executive Office of the President 2016); Ramnath Balasubramanian, Ari Libarikian and Doug McElhaney, 'Insurance 2030-The impact of Al on the future of insurance' (McKinsey, 2018) <https://www mckinsey.com/industries/financial-services/our-insights/insurance -2030-the-impact-of-ai-on-the-future-of-insurance> accessed 10 July 2018. 
Another very relevant area of application is medicine. In 2016, a paper in JAMA by Gulshan and colleagues showed that an AI system was able to identify diabetic retinopathy and diabetic macular oedema in retinal fundus images with a degree of accuracy similar to that of licensed ophthalmologists. ${ }^{5}$ One year later, on the same journal, a research group form the Netherlands demonstrated that an AI system employing deep learning (a form of machine learning) is able to detect nodal metastases in women with breast cancer with the same accuracy as clinical pathologists. ${ }^{6}$ A medical AI system that interprets magnetic resonance images in cardiology and radiology has already been licensed for use. ${ }^{7}$ Moreover, earlier this year, the FDA gave clearance to an AI system that helps orthopaedists detect wrist fractures in twodimensional x-ray scans. ${ }^{8}$

While most people recognize the promise of applying AI systems to medical diagnosis and decisionmaking, many are worried about the use of partly autonomous computer programs for medical purposes. This fear has to do with a characteristic of many ML methods. AI systems that incorporate ML learn with a varying degree of supervision which rules ${ }^{9}$ they need to follow in order to perform their task. The programmer sets up the system so that it can learn to do something. However, he or she does not decide, nor is necessarily aware of the rules the AI system has learnt and is following in order to do what it is supposed to do. This characteristic is often referred to as the opacity of ML. For the same reason, AI systems based on ML are often called black boxes, to stress that it is hard or even impossible for human users to open them up, so to say, and see for themselves what the machine is doing (or, which is the same, what rule the machine has learnt and is employing). The possibility that these systems could remain opaque to their own creators as well as to their end-users is a cause of concern. ${ }^{10}$

The issue of opacity in AI systems for medical applications is only starting to be discussed. For example, Char and colleagues have recently argued that pressing ethical challenges loom large on the horizon of ML in healthcare, precisely because of opacity. ${ }^{11}$ In particular, they point out that physicians lack adequate education to understand the construction and limitations of such systems and they stress that: 'Remaining ignorant about the construction of machinelearning systems or allowing them to be constructed as black boxes could lead to ethically problematic outcomes.'.

The debate about lack of algorithmic transparency has taken several directions. Some scholars have argued for a presumed 'right to explanation' for data subjects whose data is processed by means of AI systems. According to this alleged right, when profiling or automated decisions affect peoples' capacity to access certain goods and services, data subjects have a right to be provided with adequate explanations regarding the processes that led to those outcomes. The idea of a right to explanation stems from the value of transparency in data processing and it is intended to counterbalance the opacity of automated systems. However, its actual definition and scope are contested. Some of its proponents - like Wachter, Mittelstadt and Floridi - distinguish between the ex-

5 Varun Gulshan and others, 'Development and validation of a deep learning algorithm for detection of diabetic retinopathy in retinal fundus photographs' (2016) 316 Jama 2402.

6 B Ehteshami Bejnordi and others, 'Diagnostic assessment of deep learning algorithms for detection of lymph node metastases in women with breast cancer' (2017) 318 JAMA 2199.

7 Bernard Marr, 'First FDA approval for clinical cloud-based deep learning in healthcare' Forbes

8 FDA, 'FDA permits marketing of artificial intelligence algorithm for aiding providers in detecting wrist fractures' (FDA, 2018) $<$ https://www.fda.gov/NewsEvents/Newsroom/ PressAnnouncements/ucm608833.htm> accessed 29 May 2018.

9 We use the term 'rule' in a general sense, and not as commonly used in the Al field, as for instance in the case of a 'rule-based expert system'. Therefore, throughout this paper, with the word 'rule' we refer to the operations the system undertakes in order to perform a task. In the case of Al systems employing ML algorithms, such 'rules' are autonomously learnt by the system either with or without human supervision.

10 Mike Rawson, 'For artificial intelligence to thrive, it must explain itself' (The Economist, 15 February 2018) <https://www .economist.com/science-and-technology/2018/02/15/for -artificial-intelligence-to-thrive-it-must-explain-itself $>$; Niall Ferguson, 'The more machines learn, the less we shall grasp' (The Times, 11 February 2018) <https://www.thetimes.co.uk/article/ the-more-machines-learn-the-less-we-shall-grasp-x8kzddhxb> accessed 13 July 2018; Will Knight, 'The Dark Secret at the Heart of $\mathrm{Al}^{\prime}$ (MIT Technology Review, 11 April 2017) <https:// www.technologyreview.com/s/604087/the-dark-secret-at-the -heart-of-ai/> accessed 10 July 2018; Cliff Kuang, 'Can A.I. Be Taught to Explain Itself?' (The New York Times Magazine, 21 November 2017) <https://www.nytimes.com/2017/11/21/ magazine/can-ai-be-taught-to-explain-itself.html> accessed 10 July 2018.

11 Danton S Char, Nigam H Shah and David Magnus, 'Implementing machine learning in health care-addressing ethical challenges' (2018) 378 The New England journal of medicine 981.

12 ibid 983 
planation of a system's general functionality, and the explanation of specific decisions taken through or by an artificial intelligence system. ${ }^{13}$ According to this group of authors, explaining an automated decisionmaking system's functionality means explaining its 'logic, significance, envisaged consequences, and general functionality.' ${ }^{14}$ On the other hand, explaining specific decisions means explaining 'the rationale, reasons, and individual circumstances of a specific automated decision, eg the weighting of features, [or] machine-defined case-specific decision rules.'. ${ }^{15}$ According to Wachter and colleagues, only the latter form of ex post explanation of specific decisions genuinely fulfils the idea of a right to explanation. This understanding of the right to explanation has been fiercely criticised. Selbst and Powels, for instance, have argued that such framework overlooks the fundamental characteristics of AI systems. ${ }^{16}$

Interestingly, this debate has been triggered by some provisions already present in the European Data Protection Directive ${ }^{17}$ (DPD) and now restated and expanded in the European Data Protection Regulation $^{18}$ (GDPR). The GDPR establishes principles, obligations and rights in the context of profiling and automated individual decision-making. Some of these new provisions entitle data subjects to receive information, explanation and protection (in the forms of rights and safeguards) regarding profiling, automated decisions, and special categories of data involved in such activities. In particular, data subjects are entitled to receive meaningful information about the logic involved, the significance and the envisaged consequences of solely automated individual deci-

13 Sandra Wachter, Brent Mittelstadt and Luciano Floridi, 'Why a right to explanation of automated decision-making does not exist in the general data protection regulation' (2017) 7 International Data Privacy Law 76

14 ibid 78.

15 ibid 78.

16 Andrew D Selbst and Julia Powles, 'Meaningful information and the right to explanation' (2017) 7 International Data Privacy Law 233, 239.

17 Directive 95/46/EC of the European Parliament and of the Council of 24 October 1995 on the protection of individuals with regard to the processing of personal data and on the free movement of such data.

18 Regulation (EU) 2016/679 of the European Parliament and of the Council of 27 April 2016 on the protection of natural persons with regard to the processing of personal data and on the free movement of such data, and repealing Directive 95/46/EC (General Data Protection Regulation), OJ 2016 L 119/1 ('GDPR'). sion-making and profiling activities as stated in $\mathrm{Ar}$ ticles 13(2)(f), 14(2)(g) and 15(1)(h) of the GDPR.

Some scholars - including Selbst and Powels have recognized in these provisions the implementation of a right to explanation. ${ }^{19}$ Others instead like Wachter and colleagues - do not think that existing provisions in the GDPR adequately address the full scope of this right. ${ }^{20}$ It is certainly true that the GDPR mentions 'explanation' only once, in Recital 71 - and that recitals are non-binding. Yet, while the GDPR does not explicitly refer to the issue of opacity, nor to the metaphor of the black-box, it nonetheless specifies a set of rights, safeguards and conditions that require data controllers to communicate some relevant features of profiling and automated data-processing systems to data subjects. ${ }^{21}$ These provisions, apply also to AI systems in the domain of healthcare, and therefore they oblige data controllers (in this case, hospitals and physicians) to provide meaningful information to patients about the use of such systems. Yet, the implementation of such provisions in the clinical setting calls for a deeper analysis of what opacity of AI systems amounts to and how data controllers can fulfill the demands for greater transparency towards a specific category of data subjects - a category that, in this case, will likely correspond to patients. This analysis is intended to dispel some of the potential sources of confusion that may have affected current debates on the right to explanation. This will in turn facilitate the legal interpretation of the GDPR provisions on profiling and automated decision-making in the domain of healthcare, hopefully fostering consensus on efficient and yet meaningful ways to fulfil data con-

19 Bryan Goodman and Seth R Flaxman, 'European Union regulations on algorithmic decision-making and a "right to explanation" (2017) 38(3) Al Magazine 50; Selbst and Powles (n 16).

20 Wachter, Mittelstadt and Floridi (n 13).

21 We therefore concur with Selbst and Powels that the GDPR endorses the principle that data subjects are entitled not only to be informed but also to receive some kind of meaningful explanation about the use of profiling and automated data-processing. This does not mean, however, that the scope of such demanded explanations is sufficient to dispel all types of opacity in the case of $\mathrm{ML}$ algorithms. In Section III, we argue that while the GDPR establishes the principle that some information about automated data processing and resulting decisions must be provided to data subjects, not all the sources of opacity are equally amenable to be removed. We also highlight that the rights and interests of data subjects (that in our case coincide with patients) are not equally affected or called into question by all forms of opacity. Therefore, while patients need transparency about automated data processing activities, they may not need a full disclosure of the inner workings of Al systems employed in the context of healthcare. 
trollers' obligations in this rapidly evolving area of medical technology.

In what follows we will first summarise the GDPR provisions in relation to profiling and automated decision-making (II), focusing on rights and obligations regarding communication to data subjects about such activities. We then provide an analysis of the notion of opacity, its sources and how the GDPR provisions could be fulfilled in the clinical setting (III). We then discuss broader ethical and practical implications of such obligations in the context of the patient-doctor relationship (IV).

\section{Promoting Transparency: What Does the GDPR Demand}

The new GDPR is driven by the general aim of improving the protection of data subjects. ${ }^{22} \mathrm{~A}$ further aim of the Regulation is that of enhancing overall transparency of data processing activities. ${ }^{23}$ The principle of transparency is defined as requiring that any information and communication relating to the processing of ... personal data be easily accessible and easy to understand, and that clear and plain language be used.. ${ }^{24}$ In particular, data subjects should be informed about the identity of data controllers and the aims of data processing, they should obtain confirmation and communication of data processing activities employing their data and should be made aware of their rights. Further safeguards should be in place

\footnotetext{
GDPR, recital 1.

ibid, recital 39.

ibid, recital 39

ibid, recital 39.

26 The GDPR introduces or reaffirms a series of rights for data subjects, namely: the right to access (art 15), right to rectification (art 16), right to erasure (art 17), right to restriction of processing (art 18), right to data portability (art 20), right to object and automated individual decision-making (arts 21 and 22); ss 1 to 5 of ch IV specify the obligations and requirements for data controllers and processors (arts 24 to 43 ).

27 GDPR, art 4(4) (emphasis added).

28 However, it has to be stressed that other forms of data processing not employing $\mathrm{Al}$ could also amount to profiling according to the above definition.

29 Article 29 Data Protection Working Party, 'Guidelines on Automated individual decision-making and Profiling for the purposes of Regulation 2016/679' (2017) WP251 rev.01 ('Guidelines')

32 GDPR, arts 5-s and 13-21.
}

30 ibid 7 .

31 ibid 5 . to limit data collection and use only the strict minimum amount of data, to ensure privacy and to offer the possibility of rectifying or deleting data. ${ }^{25}$ These general principles apply to all kinds of data processing activities, and underlie a number of rights, obligations and requirements. ${ }^{26}$ However, special provisions exist that define rights and obligation in the case of profiling and automated decision-making. Those parts of the GDPR are of direct relevance to our present analysis, since AI systems are most likely going to fall within this type of data processing activities. The GDPR defines profiling as

any form of automated processing of personal data consisting of the use of personal data to evaluate certain personal aspects relating to a natural person, in particular to analyse or predict aspects concerning that natural person's performance at work, economic situation, health, personal preferences, interest, reliability, behavior, location or movement. $^{27}$

Automated decision-making can take place by means of profiling or not; similarly, profiling can happen with or without automated decision-making. Moreover, automated decision-making can be made with or without human involvement - in the latter case it amounts to what the GDPR defines as solely automated decision-making.

The use of AI systems for health-related purposes clearly falls under these definitions, and therefore counts as a form of profiling within the GDPR. ${ }^{28}$ This is confirmed by the Data Protection Working Party Guidelines on automated decision-making and profiling explaining the GDPR provisions in this specific domain. ${ }^{29}$ The guidelines explicitly state that profiling is generally employed to make predictions about people, using data from various sources to infer something about an individual. ${ }^{30}$ Moreover, the Guidelines note that profiling and automated decision-making can pose significant risk to the data subjects since 'these processes can be opaque.' ${ }^{31}$ This reference to that highly debated characteristic of AI systems indicates the attention of the legislators to the effects of opacity on the rights and interests of data subjects.

The specific focus on profiling and automated decision-making in the GDPR gives rise to a rather complex set of provisions that we will now briefly illustrate. We focus mainly on provisions prescribing communication and explanations of profiling, ${ }^{32} \mathrm{de}-$ 
cisions made by a human based on profiling, ${ }^{33}$ and solely automated decision-making. ${ }^{34}$

As it is customary, we will start this overview from the indications contained in non-binding recitals. ${ }^{35}$ Recital 63 states that 'every data subject should ... have the right to know ... the logic involved in any automatic personal data processing and, at least when based on profiling, the consequences of such processing. ${ }^{36}$ Recital 71 , in a similar vein, stresses that legally authorised profiling and solely automated decisions can only take place if data subjects 'right ... to obtain an explanation of the decision reached after such assessment' is respected. ${ }^{37}$

These indications clearly recall the need to counterbalance the opacity of certain forms of data processing like AI systems and to promote transparency around activities such as profiling, decision-making based on profiling and solely automated decisionmaking producing legal effects or similarly significant consequences for data subjects. ${ }^{38}$

As far as binding provisions are concerned, Article 5(1)(a) establishes transparency as a basic principle of data processing (along with lawfulness and

33 ibid, arts 5-s and 13-21.

34 ibid, art 22.

35 In EU legislation, recitals, albeit not binding per se, provide indications about how to the subsequent articles have to be interpreted and what they aim to realize.

36 GDPR, recital 63.

37 ibid, recital 71.

38 ibid, art 22(1).

39 Other general principles applying to data processing of personal data, and therefore also to profiling an automated decisionmaking include purpose limitation (ie data can be used only for purposes coherent with the original purpose of collection, art 5(1)(b)), data minimisation (art 5(1)(c)), accuracy (art 5(1)(d)), storage limitation (art 5(1)(e)), data security (art 5(1)(f)), and accountability (art 5(2)).

Informed consent by data subject can be a lawful condition for data processing activities, including profiling and non-solely automated decision making (art 6(1)(a)). The Guidelines, again, link this requirement to opacity (page 13). Since data processing can rely on data not directly provided by the data subject, inform consent, is a way to inform people about an envisaged activity regarding him- or herself and its foreseeable consequences (ibid). Consent should thus be considered a sufficient but not necessary condition for lawful data processing. Other equally sufficient conditions include: processing is necessary for the performance of a contract (art 6(1)(b)); processing is needed to comply to legal obligations $(\operatorname{art} 6(1)(\mathrm{c}))$; vital interests are at stake (art $6(1)(d)$ ); processing is in the public interest or needed to exercise official authority (art 6(1)(e)); processing is necessary to fulfil a legitimate interest (art 6(1)(f)).

40 Whenever their data are collected, according to art 13(1) and (2), data subjects have a right to receive specific information about: fairness). As explained by the Guidelines, this principle is especially relevant to profiling because this type of activity is often invisible to data subjects, but also because data subjects may not have the necessary capacities to understand the technical aspects of profiling and automated decision-making activities - which represents, as we will see below, two distinct ways of articulating opacity. ${ }^{39}$

The GDPR recognizes specific rights to data subjects that derive from the general principle of transparency. They apply to all data processing activities and articulate precise requirements concerning what data controllers are supposed to communicate to data subjects regarding the activities they intend to conduct or are conducting with their data. ${ }^{40}$

Some communication-related requirements apply exclusively to solely automated decision-making and profiling activities. Overall, data subjects have a 'right not to be subject to a decision based solely on automated processing, including profiling, which produces legal effects concerning him or her or similarly significantly affects him or her'.41 This type of activity is in principle prohibited by Article 22(1) but data controllers; the purposes of processing; the location of processing; the legitimate interests of data controllers; the recipients of their data; the duration of processing; their rights; whether data processing is a statutory or contractual requirement, is needed to enter a contract, or is mandatory (and the possible consequences of not providing data). When data are not obtained directly by the data subject, information should also include: the categories of data concerned and the source of the personal data (art 14(1) and (2)). Additionally, data subjects have a right to access their collected data at any moment (art 15(1)). Data subjects also have a right to rectify their data (art 16), to have them erased (art 17), and to restrict the scope of processing (art 18). Furthermore, according to art 21(1), data subjects have a right to object to automated individual decision-making, including profiling in the case of direct marketing (art 21(2), art 21(3)), but also in the case processing by public authorities or by third parties unless the controller demonstrates compelling legitimate interests that override data subjects' rights and freedom (as specified in art 6(1)(e) and 6(1)(f)). The same art 21, on para 6 specifies that data subjects can also object to processing in the context of research unless there are public interest reasons to continue processing their data (art 21(6)).

41 GDPR, art 22(1). The Guidelines specify that a legal effect occurs if a decision affects a person's legal rights or status, or rights that emerge from a contract (Guidelines 21). According to the Guidelines 'similarly significant' effect is one that is of 'sufficiently great or important' (Guidelines 21). More concretely, they state that a 'decision must have the potential to: significantly affect the circumstances, behaviour or choices of the individuals concerned; have a prolonged or permanent impact on the data subject; or at its most extreme, lead to the exclusion or discrimination of individuals' (Guidelines 21). Processing of health data or of other personal data in the context of healthcare arguably produces an effect of this sort, since, at a minimum, it affects her circumstances and most likely her choices in a significant or otherwise important way. 
exceptions apply as per Article 22(2): (a) when the processing is needed to enter or perform a contract between data subjects and data controllers; (b) when it is authorised by the Union or a Member State; and (c) when data subjects express explicit consent.

When special categories of data are employed in solely automated decision-making or profiling, exceptions (a) and (c) do not apply, unless the data subjects have explicitly consented to the use of such data, or processing is justified by a substantial public interest. ${ }^{42}$ Under special categories, the GDPR lists personal data

revealing racial or ethnic origin, political opinions, religious or philosophical beliefs, or trade union membership, and the processing of genetic data, biometric data ... , data concerning health or data concerning a natural person's sex life or sexual orientation. ${ }^{43}$

On the basis of this definition, solely automated decision-making and profiling in the context of medicine by means of AI systems amount to activities employing special categories of data.

As far as communication to data subjects is concerned, a data controller should inform them of the existence of solely automatic decision-making and profiling activities, and should provide 'meaningful information about the logic involved, as well as the significance and the envisaged consequences of such processing for the data subject' ${ }^{\prime 4}$ The same applies to activities based on data not obtained directly from

42 ibid, art 22(4).

43 ibid, art 9(1).

44 ibid, art 13(2)(f).

45 ibid, art 14(2)(g).

46 ibid, art 15(1)(h); Guidelines 17.

47 ibid, art. 13(2)(f); ibid, art 14(2)(g); ibid, art 15(1)(h).

48 Frank Pasquale, The black box society: The secret algorithms that control money and information (Harvard University Press 2015).

49 Another extensive and similarly oriented analysis of the opacity concept is provided also by Burrel, who defines opacity either as intentional corporate or trade secrecy, as technical illiteracy, or as the way in which algorithms operate at the scale of application.

50 Article 29 Data Protection Working Party 5. The same articulation of opacity appears on page 9: 'profiling is often invisible to the data subject

51 ibid 13.

52 W Nicholson Price II, 'Black-box medicine' (2015) 28 Harv JL \& Tech 419 . the data subject. ${ }^{45}$ Data subjects also have a right to access all their personal data used in the context of such activities, as well as all information relative to profiling itself, that is, the categories used to set up the profiling and the classifications applied to the data subject as a result of the profiling activity. ${ }^{46}$

The communication of the logic involved, [and of] the significance' of solely automated data processing brings up the issue of explainability. ${ }^{47}$ What does it mean to inform a data subject about the logic and significance of an AI system, especially using ML, in the context of healthcare? Does opacity stand in the way of fulfilling these requirements? And if so, in which specific way? To address these questions, in the next section we offer a conceptual analysis of the notion of opacity with the aim of clarifying its implications in the contest of the GDPR.

\section{Unpacking the Notion of Opacity}

The idea that AI systems can be opaque black boxes, and that this characteristic poses risks to humans who are affected by their decisions has recently gained traction. Frank Pasquale, in his 2015 book The Black Box Society, was among the first to popularise the idea that AI systems are 'black-boxes'. ${ }^{48}$ According to Pasquale, AI systems deserve this label because they pervasively collect and compute personal data to help companies pursue their business aims, while most data subjects are unaware such activities are even taking place. This may be the case because firms intentionally conceal their profiling activities in order to avoid objections on the part of data subjects or because they want to prevent competitors from becoming aware of their trade secrets. ${ }^{49}$ This view articulates a first, very common understanding of opacity, that we found also in the Guidelines to the GDPR stating that individuals might not know that they are being profiled" 50 and that they may not know that data not provided by themselves can also be used to profile them. ${ }^{51}$ We call this form of opacity 'lack of disclosure'.

The notion of opacity however possesses also other semantic connotations. For instance, the image of a black box has been extended to the application of AI systems in medicine. ${ }^{52}$ Price describes what he calls 'black-box medicine' as a form of medicine heavily based on algorithms that can analyse large amounts of data. Black box medicine relies on 
opaque algorithms to find patterns among patient data that can, for instance, make predictions about health-related risks, or suggest a better dosage of a drug. For example, one can imagine the case of a woman living in a specific urban environment and presenting a specific combination of genetic variants. A ML-based AI system may process such data and predict that the patient has, say, a higher than average risk of developing aggressive breast cancer. Price argues that such an algorithm can be opaque to the data subject, as well as to the physician, in two respects. On the one hand, the ML system may discover patterns within such a high number of variables that it is extremely hard for a human mind to make sense of. This type of opacity is the result of the sheer complexity of data computation - relying in our case on millions of genetic and environmental factors interacting with each other. We can refer to it as 'epistemic opacity' - to stress that opacity here originates from a lack of understanding regarding how the ML system operates. On the other hand, opacity may amount to the fact that, while an ML system can identify patterns in an incredibly high number of variables, it may not be possible to trace them back to any known causal explanation of the association between input and output, that is, between patient data and the prediction made by the system. In other words, since the ML system is not programmed with any particular clinical hypothesis in mind (eg the hypothesis that certain genetic mutations increase the risk of breast cancer), it may discover patterns that, at the present stage of medical knowledge, cannot be linked to any known causal explanation. We can call this kind of opacity 'explanatory opacity', to stress that it refers to the lack of a clinical explanation or, that is the same thing, to the impossibility of clinically interpreting the outcome of the ML system.

This rapid excursus shows that there are at least three different ways of interpreting the meaning of opacity: as lack of disclosure, as epistemic opacity, or as explanatory opacity. ${ }^{53}$ These three semantic dimensions of opacity are particularly relevant to the application of AI systems in medicine. However, this conceptual framework can be fruitfully applied to the any other use of AI systems that fall under the GDPR provisions.

In what follows we will discuss each of these three semantic dimensions of opacity in the context of the requirements, rights and obligations stipulated by the GDPR in relation to automated decision-making and profiling activities.

\section{Lack of Disclosure}

From a general point of view, lack of disclosure refers to the fact that data subjects are unaware that automated decision-making and profiling activities about them are being carried out. This type of opacity does not depend on intrinsic technical characteristics of AI systems, but derives from the way automated data processing and profiling activities are conducted. In principle, any type of data processing activity could be conducted without the data subjects being aware of it. It follows that lack of disclosure is not specific to the use of AI systems. Yet in the field of automated decisions and profiling, this kind of opacity prevents data subjects from exercising some specific data-related rights, such as the right to object to solely automated data processing as stated in Article 13(2)(f). This may have very tangible consequences in the context of medical applications.

Lack of disclosure may depend on intentional concealment of relevant information regarding automated data processing. This may be linked to the attempt on the part of data controller to avoid interference with or potential objections to their activities, but it can also be due to the need to protect intellectual property, copyright and trade secrets. Since algorithms and data are non-rival goods, once disclosed, their value can be dramatically reduced unless protected through patents. Therefore, there is lack of transparency when AI systems are subject to non-disclosure policies. Particularly, private corporations might try to use trade secrets to protect against competitors. Recital 63, although not legally enforceable, affirms that data subjects should have the right to know 'the logic involved in any automatic personal data processing and, at least when based on profil-

53 Diakopulous defines algorithmic opacity as both a lack of explanation and clarification of the $\mathrm{Al}$ system outcomes, as well as an extreme level of technical complexity that obfuscates the understanding of an Al system's working process (see Nicholas Diakopoulos, 'Algorithmic Accountability' (2015) 3(3) Digital Journalism 398). Finally, a group of researchers at Harvard differenti ate between a type of opacity described as the unawareness of signals flowing through an Al system, and a type of opacity that consists in how certain factors were used by the Al to define a specific outcome (see Finale Doshi-Velez and others, 'Accountability of Al under the law: The role of explanation' arXiv preprint arXiv:171101134). 
ing, the consequences of such processing,. ${ }^{54}$ This information shall be disclosed to data subjects pursuant Articles 13 and 14 on information, and Article 15 on data access. Yet, the same Recital 63 recognizes that such disclosure shall not adversely affect trade secrets, intellectual property and copyrights on software. It follows that, while data controllers must disclose that they are conducting profiling or automated data processing, they are not obliged to reveal all details about their AI systems. In practical terms, this entails that data controllers may still be required to provide information regarding the general characteristics of their system, but they may not be compelled to explain what rules the AI system follows, how it has reached a conclusion, or how it has taken a given decision about a particular data subject.

Lack of disclosure may also depend on the fact that the data controller is employing data not directly provided by data subjects, but inferred from other data available to the data controller. In this case, the data subject will most likely remain unaware of profiling activities about him or herself unless the data controller communicates details about such activities. Now, according to the GDPR, data subjects have a right to receive a number of information regarding who is processing their data, for which purposes and under which circumstances. ${ }^{55}$ Yet data controllers are not obliged to disclose the technical nature of their data processing activities. If, however, data processing amounts to solely automated processing ${ }^{56}$ producing legal effects or similarly significant consequences, the Regulation stipulates that they should

\footnotetext{
54 GDPR, recital 63.

55 ibid, arts 13, 14 and 15.

56 ibid, art 22(1).

57 ibid, arts 13(2)(f) and 15(1)(h).

58 ibid, art 14(2)(g).

59 ibid, arts 13 and 14.

60 Article 4(4) of the GDPR: " profiling" means any form of automated processing of personal data consisting of the use of personal data to evaluate certain personal aspects relating to a natural person, in particular to analyse or predict aspects concerning that natural person's performance at work, economic situation, health, personal preferences, interests, reliability, behaviour, location or movements;'.

61 See s IV

62 Informed consent is anyway mandatory for solely automated employing special types of data, including health data, as specified in art 9 of the GDPR.

63 Effy Vayena and Alessandro Blasimme, 'Biomedical Big Data: New Models of Control Over Access, Use and Governance' (2017) 14 Journal of Bioethical Inquiry 501.
}

inform data subjects about or confirm the existence of such form of processing, and provide 'meaningful information about the logic involved, as well as the significance and the envisaged consequences of such processing for the data subject. ${ }^{\prime 5}$ This applies also to uses of data that have not been directly obtained from data subjects. ${ }^{58}$ This type of information should happen already at the moment of data collection. ${ }^{59}$ However, such provisions do not apply to non-solely automated profiling as defined in Article $4 .{ }^{60}$ It follows that data controllers are not obliged to communicate the existence, the logic and the foreseeable consequences of their automated decision-making and profiling activities if they include even a minimal degree of human involvement.

In the medical context, this form of disclosure appears all the more important given the rapid growth of AI-based automated decision-making tools in the practice of medicine. In this case, disclosure other than being legally mandatory, is also justified by the need to promote patients' trust in the use of AI systems in healthcare. Failing to reveal to the patient that such systems are in use may undermine the fiduciary relationship between patients and doctors, and may also give patients the impression that they are being marginalized in decisional processes regarding their health, thus affecting their decisional autonomy and their sense of self-determination. In light of these considerations, restricting disclosure to solelyautomated activities may turn out to be insufficient. ${ }^{61}$ Informed consent seems to be the most obvious way to discharge such informational obligations regarding the clinical use of AI systems ${ }^{62}$. However, research is needed to show whether conventional informed consent procedures do actually constitute a valid means to convey this type of information and to increase patients' trust. Other means of communication, including innovative forms of consent ${ }^{63}$ may actually prove equally or more effective than conventional written consent.

\section{Epistemic Opacity}

Epistemic opacity occurs when it is not possible to have access to or there is not sufficient understanding of the rules an AI system is applying to make predictions, classifications and decisions. Epistemic opacity is therefore related to the question of how an AI system provides a specific outcome. 
This type of opacity can have two sources: a) procedural darkness or b) procedural ignorance.

\section{a. Procedural Darkness}

Procedural darkness occurs when the developers or the users themselves (and as a consequence, data subjects) do not have access to the rules that the AI system is following to produce a certain output. This is a consequence of how ML systems work. A ML system can learn the rules that it will apply to make classifications and decisions based on given input data. This means that neither the developer, nor the user (eg a physician or a healthcare provider) is aware of how the system produces its outputs. This information is usually encoded in an abstract form in the parameters that the ML system has learnt and therefore it is not readily accessible in a semantically readable form. As a consequence, programmers and users may not be in a position to disclose meaningful information about the way a given output has been reached. While this does not prevent data controllers from explaining the general working principles of the systems, it limits their capacity to reveal exactly which features of the input data have been taken into account to arrive at a given decision about a given data subject. This does not mean, however, that such explanation is impossible, and that this type of opacity cannot be dispelled - and actually the GDPR does not require this type of explanation. Moreover, it is unclear whether the patients' rights and interests are truly promoted by getting access to this type of information.

\section{b. Procedural Ignorance}

Even assuming that the rules of an AI system are accessible in a semantic form, acquiring a meaningful understanding of their role in data processing activities may require a considerable amount of background knowledge. In the case of AI systems used in oncology for instance, a patient should possess basic notions of both cellular pathology and computer science to make sense of what an AI system for automated pathology screening does when it looks at scanned images of human biopsies. In the presence of procedural ignorance, the patient cannot grasp information about the rules the AI system is following. This can also happen because such rules are simply too many. The same problem can occur with attempts at explaining which rules the system applies in his or her specific case. Moreover, it cannot be assumed that physicians possess enough insight into these matters either. Patient education sessions and specifically trained consultants could be helpful to dispel procedural ignorance. In general, however, it remains to be seen whether dispelling procedural ignorance is indeed needed to ensure patients meaningfully consent to the use of AI systems in healthcare. The GDPR seems more oriented to explanations that do not dwell into the technical details of automated data processing and profiling activities, but rather provide accessible yet meaningful explanation of the general principles involved in their design. ${ }^{64}$ The aim of such provision, seems linked to the possibility of exerting the rights that the Regulation recognizes to data subjects, including the right to object to fully automated data processing and profiling.

Epistemic opacity limits the possibility of providing thorough explanations of either the inner workings of AI systems or the specific rules used to make a specific, individual decision. However, these limitations do not entail the impossibility of explaining the foreseeable consequences of such processing. ${ }^{65}$ Moreover, according to the Guidelines, data subjects who are the object of profiling activities are still entitled to access the 'details of any personal data used for profiling, including the categories of data used to construct a profile ... and details of which segments the data subject has been placed into'.66

AI systems can operate on a too complex level of inputs to be understandable by humans. For example, machine learning is able to predict whether a patient has diabetes ${ }^{67}$ or lung cancer ${ }^{68}$ including hundreds of thousands of heterogeneous data (eg from images, personal health records, lifestyle data collected through wearables devices and so on) in multiple combinations. ${ }^{69}$ Medical algorithms can also make predictions about the response of a tumour to a spe-

64 GDPR, arts 13, 14 and 15.

65 ibid, arts 13(2)(f), 14(2)(g) and 15(1)(h).

66 Article 29 Data Protection Working Party 17.

67 Gulshan and others (n 5).

68 Kun-Hsing Yu and others, 'Predicting non-small cell lung cancer prognosis by fully automated microscopic pathology image features' (2016) 7 Nature communications 12474

69 Ziad Obermeyer and Ezekiel J Emanuel, 'Predicting the future-big data, machine learning, and clinical medicine' 375 The New England journal of medicine 1216. 
cific drug looking at allelic patterns among thousands of genes. ${ }^{70}$ Yet, prognostic models commonly used by physicians until recently were restricted only to a relatively limited number of variables. ${ }^{71}$ Even in the case in which the physician could in principle understand the datasets and the rules used by the machine, complexity may still hinder his or her full understanding of how the system operates. The information available to the physician would be either too large in volume or computed in such an elaborated way to be nearly impossible to grasp at a cognitive level. Neither the doctor nor an expert of AI systems might be able to understand in detail how the data is processed exactly and how the output is computed from the data. ${ }^{72}$

It should be noted, however, that AI specialists are trying to reduce procedural darkness by technical means. Different techniques allow deeper insights into AI systems. In image recognition, for example, a method called deconvolution provides information about what rules very complex systems like artificial neural networks learn. ${ }^{73}$ Deconvolution allows to visualise otherwise internal - and inaccessible - states of the system but the rules the system uses for its decision-making have to be derived experimentally. Such a method could lead researchers to realize that, for instance, some particular properties of an image (eg shapes, edges, colours) are what the system is looking at to determine the outcome. While this is a very interesting area of research, it remains to be seen whether and how such knowledge could one day be employed to provide more detailed explanations to data subjects. In the case of clinical applications, then, these considerations will also need to take into ac-

70 W Nicholson Price II, 'Medical Malpractice and Black-Box Medicine' Health Law, and Bioethics (Cambridge University Press, Forthcoming).

71 ibid.

72 Jenna Burrell, 'How the machine "thinks": Understanding opacity in machine learning algorithms' (2016) 3 Big Data \& Society 1.

73 Matthew D Zeiler and Rob Fergus, Visualizing and understanding convolutional networks (Springer 2014).

74 As Hildebrandt (see Mireille Hildebrandt, 'Defining profiling: a new type of knowledge?' in Profiling the European citizen (Springer 2008)) has explained, 'correlations stand for a probability that things will turn out the same in the future. What they do not reveal is why this should be the case' (see Hildebrandt, page 18). The use of algorithmic decisions in an increasingly wider range of applications has led some (see Hildebrandt, eg page 27) to caution against the rise of a 'black box' society and demand increased transparency in algorithmic decision-making.

75 Peter Machamer, Lindley Darden and Carl F Craver, 'Thinking about Mechanisms' (2000) 67 Philosophy of Science 1. count the specific clinical circumstances at stake, as well as the best interests of the patient.

\section{Explanatory Opacity}

Explanatory opacity relates to the question of why an AI system provides a specific outcome. What an ML system is designed to do is to discover patterns between huge numbers of variables in a training dataset, and to leverage these patterns to make classifications, predictions and decisions regarding new input data. The output, in other words, is the result of patterns that the ML system has generalised from training examples. In the field of medicine, for example, an ML system could learn that certain geometrical properties of a histology slide correlate with a bad prognosis. In an extremely simplified scenario, we can imagine that the system learns that a given cellular shape is linked to a bad prognosis. It may well be that this rule corresponds exactly to one of the criteria that pathologists use to recognize an aggressive tumour - and therefore to adequately predict a bad prognosis. Pathologists may also have a scientific explanation of the reason why aggressive tumour cells acquire that shape and behave the way they do. It could be, however, that the ML system looks at properties like relative pixel luminosity that human pathologists do not use, and whose connection to a given clinical phenotype is not known. In those cases, one could confidently say that there is a statistically relevant correlation between the property and the clinical classification. Still, one would not have a scientific explanation of the reason why the property and the classification are linked one to the other. ${ }^{74}$ In order to possess such an explanation, one should know the biological mechanism that connects the observed property with the predicted phenotype, or, in other words, one should know a causally relevant chain of biological facts underlying the association between the two. ${ }^{75}$ We have called the absence of this kind of knowledge 'explanatory opacity'.

This characteristic is typical but not exclusive of knowledge produced through ML systems. There are in fact many examples in medicine of perfectly reliable correlations that lack a scientific explanation. For instance, it has long been known that being heterozygous for a point mutation in the $\beta$-chain of the human haemoglobin gene (a mutation that in homozygosity causes sickle cell anaemia) provides pro- 
tection against malaria. ${ }^{76}$ Yet only recently has the biological mechanism by which this mutation confers protection been elucidated. Analogously, very little is $\mathrm{known}^{77}$ about the reason why acetaminophen (paracetamol) works, and yet this is one of the most common pain killers and antipyretic drugs worldwide. Examples of other drugs currently in use despite limited knowledge regarding why they work are countless $^{78}$. This shows that explanatory opacity does not represent a dead end in the context of medicine. Little research exists however to analyse whether opaque AI systems in medicine pose specific risks to patients. Also, it is not clear whether the notion of 'meaningful information' to be communicated to data subjects in the case of automated data processing and profiling may include providing causal mechanistic explanations of algorithmic decisions. The Guidelines suggest that this is not the case, and that explanations should focus on the rationale for data processing rather than on its scientific interpretability. ${ }^{79}$

It could be argued, however, that reduced explanatory opacity constitutes a reassurance regarding the clinical validity of medical AI systems. Moreover, physicians may be more likely to adopt such systems if they could be provided with clinically intelligible reasons for using them. Yet, this does not imply that this type of information should be communicated to patients, nor that they have a right to it. It may be and more research could help find out - that patients are not interested in this type of detailed scientific explanations. Moreover, it is still not known whether this type of explanations have an impact on patients' trust in the use of these technologies. What is certainly relevant for promoting the rights and interests of patients is that AI systems used in healthcare respond to the highest standards of safety and efficacy. Careful clinical validation processes by regulatory agencies and through self-imposed industry standards will have to be adopted, and the implications of explanatory opacity should be duly taken into account in the development of such standards.

An alternative to providing in-depth information about an AI system and a way to overcome the implications of explanatory opacity lies in counter-factually examining the outcomes provided by the AI system. Wachter, Mittelstadt and Russell have argued that 'counterfactuals describe a dependency on the external facts that led to that decision' and that more than one counterfactual might exist. ${ }^{80}$ Counterfactu- als can illustrate how even a small change in an input variable can result in a different outcome. Input variables can therefore be systematically varied and corresponding outcomes can be compared to the original, in order to gain deeper insight into the relationship between input variables and the computed outcomes. This opens up the possibility to infer a reason why the original outcome was produced. The explanation provided based on a specific case might not result in an exhaustive description of the entire AI system, but it would contribute significantly to the perspective of human understanding. ${ }^{81}$

\section{Discussion}

With the rapid development of AI systems for medical use, patient data will increasingly be used in automated data processing, profiling and decision-making activities. These activities are not restricted to data types that clearly fall within the remit of health data. As a matter of fact, an increasing variety of data generated and collected outside the clinical setting, and not initially intended for medical use are now starting to be employed in diagnosis, health-risk predictive models and to guide medical decisions. ${ }^{82}$ These include, for instance, lifestyle data, data about dietary habits, socio-economic data, but also data such as keystroke dynamics, ${ }^{83}$ and in general data collected through smartphones ${ }^{84}$ or wearable de-

76 Ana Ferreira and others, 'Sickle Hemoglobin Confers Tolerance to Plasmodium Infection' (2011) 145 Cell 398.

77 Burkhard Hinz, Olga Cheremina and Kay Brune, 'Acetaminophen (paracetamol) is a selective cyclooxygenase-2 inhibitor in man' (2008) 22 The FASEB journal 383.

78 Tanya Lewis, 'Mystery Mechanisms' (The Scientist, 29 July 2016 $<$ https://www.the-scientist.com/news-analysis/mystery -mechanisms-33119> accessed 1 July 2018.

79 Article 29 Data Protection Working Party 25-26.

80 Sandra Wachter, Brent Mittelstadt and Chris Russell, 'Counterfactual Explanations Without Opening the Black Box: Automated Decisions and the GDPR' (2017) 31 Harvard Journal of Law \& Technology 6.

81 Doshi-Velez and others (n 53)

82 Effy Vayena and Alessandro Blasimme, 'Health Research with Big Data: Time for Systemic Oversight' (2018) 46 The Journal of Law, Medicine \& Ethics 119

83 Ryen W White, P Murali Doraiswamy and Eric Horvitz, 'Detecting neurodegenerative disorders from web search signals' (2018) 1 npj Digital Medicine 8.

84 Laura Weiss Roberts, Steven Chan and John Torous, 'New tests, new tools: mobile and connected technologies in advancing psychiatric diagnosis' (2018) 1 npj Digital Medicine 20176. 
vices. ${ }^{85}$ In this expanding health data ecosystem, ${ }^{86}$ new medical paradigms such as precision medicine ${ }^{87}$ and digital health ${ }^{88}$ are rapidly growing with the aim of exploiting novel capabilities in data mining and automated data processing for the benefit of patients. These developments go hand in hand with the increasingly perceived need to promote data sharing so as to accelerate the pace of progress in this domain. ${ }^{89}$ Data sharing, however, needs to be balanced against the risks that unauthorised access and misuse of personal data may pose to data subjects. The GDPR sets out to mitigate such risks regarding pervasive data processing. While health-relevant data are not the main focus of this law, its provisions clearly apply also to the use of data in the medical field. Patients therefore have additional rights as data subjects. Physicians and healthcare providers in general have specific responsibility as data controllers. Such entitlements and obligation are likely to acquire increasing importance with the rapid development and deployment of AI systems in the practice of medicine. The opacity of these systems, however, calls into question their reliability and trustworthiness. If left unattended, this issue may undermine the development of these technologies and forego the muchawaited benefit they promise to deliver to patients.

Our analysis has shown that opacity is a polysemic concept. This notion is used by different people to indicate different practical and technical characteristics of AI systems. We also showed that not all dimensions of opacity are equally amenable to be dis-

85 Gillian Gresham and others, 'Wearable activity monitors to assess performance status and predict clinical outcomes in advanced cancer patients' (2018) 1 npj Digital Medicine 27.

86 Effy Vayena and others, 'Policy implications of big data in the health sector' 96 Bulletin of the World Health Organization 66.

87 Alessandro Blasimme and Effy Vayena, 'Becoming partners, retaining autonomy: ethical considerations on the development of precision medicine' (2016) 17 BMC Medical Ethics 67; Alessandro Blasimme and Effy Vayena, "Tailored-to-You": Public Engagement and the Political Legitimation of Precision Medicine' (2016) 59 Perspectives in Biology and Medicine 172.

88 Effy Vayena and others, 'Digital health: meeting the ethical and policy challenges' (2018) 148 Swiss Medical Weekly.

89 Alessandro Blasimme and others, 'Data Sharing For Precision Medicine: Policy Lessons And Future Directions' (2018) 37 Health Affairs 702

90 M Simpson and others, 'Doctor-patient communication: the Toronto consensus statement' (1991) 303 British Medical Journal 1385.

91 Glyn Elwyn and others, 'Shared Decision Making: A Model for Clinical Practice' (2012) 27 Journal of General Internal Medicine 1361.

92 Wachter, Mittelstadt and Floridi (n 13). pelled by offering information and explanations to data subjects. However, the GDPR requirements about opacity seem not to require that data subjects are provided with extensive and detailed technical information about the working logic of AI systems processing their data. At least in this respect, the GDPR adopts a not too expansive understanding of transparency.

In the specific domain of healthcare, the amount and type of information provided to patients has long been a topic of discussion due to its practical and ethical implications. ${ }^{90}$ More research is needed to understand patients' and physicians' attitudes towards opacity in AI systems. The explainability of such systems and the obligations relative to the communication of information about them are likely to affect the patient-doctor relationship. Solely automated decisions and profiling can de-personalize such relationship, reducing occasions for direct personal interaction. Patients may feel undermined from the point of view of their decisional autonomy and capacity to influence healthcare provision practices about themselves. Reasonable communication could mitigate this effect, be a precondition for objecting to fully automated processing, and for requiring some form of human involvement in those activities. This would foster ideals of shared decision making in medicine. ${ }^{91}$ Also, evidence is needed to understand if written informed consent is the most effective way of communicating information in this domain, or whether other forms of communication could be fruitfully explored.

Another ethically relevant aspect of opaque AI systems is that they may provide predictive information that is not actionable or difficult to interpret. If AI systems create the basis for the unmediated provision of such medical information directly to patients, the information burden on patients may be disproportionately big. Ad hoc safeguards need to be in place to ensure that professional figures stay in the loop of this novel ways of producing medical information and providing healthcare. This raises a thorny issue that, to date, has not received sufficient attention. Paradoxically, according to the GDPR, if a human actor is involved in the operations of an AI system, the obligation to disclose automated data processing and to provide meaningful information about its logic, significance and consequences no longer holds. ${ }^{92}$ Therefore, it is precisely the presence of a potential intermediary professional figure that 
dilutes the requirements to disclose some specific information about how AI systems work. This would create a situation in which, for instance, developers of fully automated wellness smartphone apps, but not physicians employing clinical-grade AI systems as aid to their activity, may have to deliver information about the use of AI systems to patients. On the other hand, in the case of fully automated systems, the communication requirements of the GDPR, may also add a further burden to doctors who are already overwhelmed by bureaucratic and administrative duties.
Furthermore, it should be reminded that, while our conceptual analysis arose from newly instituted GDPR provisions, it is not limited to medicals uses of AI, but applies to AI systems in general. As AI systems make inroads into multiple areas of human activity, including medicine, ensuring transparency requires addressing the opacity of such systems. With this article, we have proposed a conceptual framework to help shed light into the much-debated issue of AI opacity and the need for more transparent and accountable uses of artificial intelligence in medicine and beyond. 